\title{
Effect of structural disorder on the Kitaev magnet $\mathrm{Ag}_{3} \mathrm{LiIr}_{2} \mathrm{O}_{6}$
}

\author{
Faranak Bahrami $\odot,{ }^{1}$ Eric M. Kenney, ${ }^{1}$ Chennan Wang, ${ }^{2}$ Adam Berlie, ${ }^{3}$ Oleg I. Lebedev, ${ }^{4}$ \\ Michael J. Graf $\odot{ }^{1}{ }^{1}$ and Fazel Tafti ${ }^{1}{ }^{1, *}$ \\ ${ }^{1}$ Department of Physics, Boston College, Chestnut Hill, Massachusetts 02467, USA \\ ${ }^{2}$ Laboratory for Muon Spin Spectroscopy, Paul Scherrer Institute, CH-5232 Villigen, Switzerland \\ ${ }^{3}$ ISIS Neutron and Muon Source, Science and Technology Facilities Council, Rutherford Appleton Laboratory, \\ Didcot OX11 0QX, United Kingdom \\ ${ }^{4}$ Laboratoire CRISMAT, ENSICAEN-CNRS UMR6508, 14050 Caen, France
}

(Received 12 November 2020; revised 20 January 2021; accepted 3 March 2021; published 17 March 2021)

\begin{abstract}
The search for an ideal Kitaev spin liquid candidate with anyonic excitations and long-range entanglement has motivated the synthesis of a new family of intercalated Kitaev magnets such as $\mathrm{H}_{3} \mathrm{LiIr}_{2} \mathrm{O}_{6}, \mathrm{Cu}_{2} \mathrm{IrO}_{3}$, and $\mathrm{Ag}_{3} \mathrm{LiIr}_{2} \mathrm{O}_{6}$. The absence of a susceptibility peak and a two-step release of the magnetic entropy in these materials have been proposed as evidence of proximity to the Kitaev spin liquid. Here we present a comparative study of the magnetic susceptibility, heat capacity, and muon spin relaxation ( $\mu$ SR) between two samples of $\mathrm{Ag}_{3} \mathrm{LiIr}_{2} \mathrm{O}_{6}$ in the clean and disordered limits. In the disordered limit, the absence of a peak in either susceptibility or heat capacity and the lack of zero-field muon precession in the $\mu$ SR signal give the impression of a proximate spin liquid state. However, in the clean limit, peaks are resolved in both susceptibility and heat capacity, and spontaneous oscillations appear in the $\mu$ SR signal, confirming long-range antiferromagnetic order in the ground state. The $\mu \mathrm{SR}$ oscillations fit to a Bessel function, characteristic of incommensurate order, as reported in the parent compound $\alpha-\mathrm{Li}_{2} \mathrm{IrO}_{3}$. Our results clarify the role of structural disorder in the intercalated Kitaev magnets.
\end{abstract}

DOI: 10.1103/PhysRevB.103.094427

\section{INTRODUCTION}

A long-standing challenge in condensed-matter physics has been to access a quantum spin liquid (QSL) ground state characterized by long-range entanglement and fractionalized anyonic excitations [1-3]. One of the most promising theoretical models of QSLs is the Kitaev model based on interacting spin- $1 / 2$ ions on a two-dimensional honeycomb lattice with bond-dependent Ising axes [4]. The prime candidates for the Kitaev model are $\alpha-\mathrm{Li}_{2} \mathrm{IrO}_{3}, \mathrm{Na}_{2} \mathrm{IrO}_{3}$, and $\alpha-\mathrm{RuCl}_{3}$, but all three compounds order magnetically at low temperatures [5-10]. Recently, a new class of intercalated Kitaev magnets was synthesized via a topochemical exchange of the interlayer $\mathrm{Li} / \mathrm{Na}$ atoms in $\alpha-\mathrm{Li}_{2} \mathrm{IrO}_{3}$ and $\mathrm{Na}_{2} \mathrm{IrO}_{3}$ with $\mathrm{H}, \mathrm{Cu}$, or $\mathrm{Ag}$ atoms, producing $\mathrm{H}_{3} \mathrm{LiIr}_{2} \mathrm{O}_{6}, \mathrm{Cu}_{3} \mathrm{LiIr}_{2} \mathrm{O}_{6}$, $\mathrm{Cu}_{2} \mathrm{IrO}_{3}$, and $\mathrm{Ag}_{3} \mathrm{LiIr}_{2} \mathrm{O}_{6}$ [11-15]. It has been claimed that this new family of Kitaev magnets, specifically $\mathrm{H}_{3} \mathrm{LiIr}_{2} \mathrm{O}_{6}$ and $\mathrm{Ag}_{3} \mathrm{LiIr}_{2} \mathrm{O}_{6}$, are closer to the QSL phase based on the absence of magnetic ordering in thermodynamic measurements, scaling behavior in the heat capacity, and a two-step release of the magnetic entropy $[11,14,16,17]$. Both bond disorder and modified interlayer coordination have been hypothesized as possible mechanisms for the proximity to the QSL ground state $[13,14,17,18]$. Currently, there is no careful experimental work to examine these hypotheses and elucidate the role of structural disorder in the intercalated Kitaev magnets.

In this paper, we present a careful study of the effect of structural disorder on one of the intercalated Kitaev magnets,

*fazel.tafti@bc.edu
$\mathrm{Ag}_{3} \mathrm{LiIr}_{2} \mathrm{O}_{6}$. We show that the signatures of magnetic ordering may be hidden in a disordered sample, but they emerge unmistakably in a clean sample. Based on our experimental results, the onset of magnetic ordering in the clean limit is unaffected by the interlayer coordination, and the nature of disorder in $\mathrm{Ag}_{3} \mathrm{LiIr}_{2} \mathrm{O}_{6}$ is inconsistent with a randomized bond picture [17]. Our experimental discussion is organized in four sections. First, in a clean sample (S1), we reveal a peak in the magnetic susceptibility at the Néel temperature $T_{N}=14 \mathrm{~K}$, followed by a sharper downturn at $T_{\mathrm{LRO}}=8 \mathrm{~K}$. Such a peak is absent in a disordered sample (S2). Second, we also reveal a peak in the heat capacity of $\mathrm{S} 1$ at $T_{N}$, which turns into a mild change in slope in S2. In light of these findings, we will revisit the two-step entropy release that has been interpreted as evidence of spin fractionalization in $\mathrm{Ag}_{3} \mathrm{LiIr}_{2} \mathrm{O}_{6}, \alpha-\mathrm{Li}_{2} \mathrm{IrO}_{3}$, $\mathrm{Na}_{2} \mathrm{IrO}_{3}$, and $\alpha-\mathrm{RuCl}_{3}[14,19,20]$. Third, using muon spin relaxation $(\mu \mathrm{SR})$ measurements, we show that $T_{N}$ marks the onset of an incommensurate magnetic order with short-range correlations that become long range below $T_{\mathrm{LRO}}$ in the clean sample, S1. The $\mu \mathrm{SR}$ oscillations are not visible in sample S2. Fourth, we use transmission electron microscopy (TEM) to reveal extended regions of silver inclusion within the honeycomb layers of S2 that are absent in S1. Complementary data and analyses are presented in four Appendices at the end.

\section{EXPERIMENTAL METHODS}

$\mathrm{Ag}_{3} \mathrm{LiIr}_{2} \mathrm{O}_{6}$ was synthesized via a topotactic cationexchange reaction as reported in Ref. [14]. To improve the sample quality, however, we took two important additional 
measures. First, we minimized the stacking faults in the precursor $\alpha-\mathrm{Li}_{2} \mathrm{IrO}_{3}$ by performing a sequential solid-state synthesis at $900^{\circ} \mathrm{C}, 1000^{\circ} \mathrm{C}$, and $1015^{\circ} \mathrm{C}$ for 24,32 , and $48 \mathrm{~h}$, respectively. Second, we increased the duration of the topotactic reaction to several days to ensure a complete exchange of the high-quality $\alpha-\mathrm{Li}_{2} \mathrm{IrO}_{3}$ precursor (see also Appendix A). Sample S1 was made with the improved technique, and sample $\mathrm{S} 2$ was made with the methods described in Ref. [14].

The electron diffraction (ED) and high-angle annular darkfield scanning TEM (HAADF-STEM) were performed using an aberration-corrected JEM ARM200F microscope. Powder x-ray diffraction was performed using a Bruker D8 ECO instrument equipped with a $\mathrm{Cu} K_{\alpha}$ source and a one-dimensional LINXEYE-XE detector. Magnetization and heat capacity were measured using Quantum Design MPMS3 and Dynacool PPMS, respectively.

The $\mu$ SR experiments were carried at the Paul Scherrer Institute (PSI) using a ${ }^{3} \mathrm{He}$ refrigerator with the Dolly Multi Purpose Surface-Muon Instrument (sample S1) and a gas flow cryostat with the General Purpose Surface-Muon (GPS) Instrument (both samples). The MUSRFIT program [21] was used for data analysis. Sample S1 was pressed into a pellet $13 \mathrm{~mm}$ in diameter and $1 \mathrm{~mm}$ thick, and sample S2 was $13 \mathrm{~mm}$ in diameter and $1.2 \mathrm{~mm}$ thick. The pellets were wrapped in a $25-\mu \mathrm{m}$ thin silver foil and mounted with varnish on copper holders. The same holder was used to mount S1 in both spectrometers. Initial measurements were made on sample S2 using a dilution refrigerator and gas flow cryostat on the EMU spectrometer at the ISIS Muon Source at the Rutherford Appleton Laboratory.

\section{RESULTS AND DISCUSSION}

\section{A. Magnetic susceptibility}

The first evidence of magnetic ordering in a high-quality $\mathrm{Ag}_{3} \mathrm{LiIr}_{2} \mathrm{O}_{6}$ sample (S1) is a peak at $T_{N}=14 \mathrm{~K}$ in the DC susceptibility $\chi$, as seen in Fig. 1(a) and magnified in Fig. 1(b). A similar behavior was observed in an earlier work by Todorova et al. [15]. The peak is broad and shows splitting between the zero-field-cooled (ZFC) and field-cooled (FC) conditions [inset in Fig. 1(a) and Appendix B]. A second temperature scale in Fig. 1(b) is $T_{\mathrm{LRO}}=8 \mathrm{~K}$, below which, the susceptibility visibly turns down (and the $\mu \mathrm{SR}$ data reveal clear oscillations in Sec. III C). Thus, we identify $T_{N}$ as the onset of short-range magnetic ordering that becomes long range below $T_{\mathrm{LRO}}$.

We compare the magnetic susceptibility of the clean sample (S1) and disordered sample (S2) in Fig. 1(c). A susceptibility peak is present in the former but absent in the latter. The absence of such a peak in a sample with the same quality as $\mathrm{S} 2$ has been misinterpreted as evidence of proximity to a Kitaev spin liquid [14]. After tremendous effort to remove disorder and improve the quality of $\mathrm{Ag}_{3} \mathrm{LiIr}_{2} \mathrm{O}_{6}$, we were able to resolve the antiferromagnetic (AFM) peak in the high-quality sample (S1). Based on our results, it would be insightful to revisit recent claims of a quantum spin liquid phase in another Kitaev material, $\mathrm{H}_{3} \mathrm{LiIr}_{2} \mathrm{O}_{6}$, which suffers from a higher disorder level than $\mathrm{Ag}_{3} \mathrm{LiIr}_{2} \mathrm{O}_{6}$ [11,22]. A large low-temperature tail in $\chi(T)$ has been observed in $\mathrm{H}_{3} \operatorname{LiIr}_{2} \mathrm{O}_{6}$ that is similar to
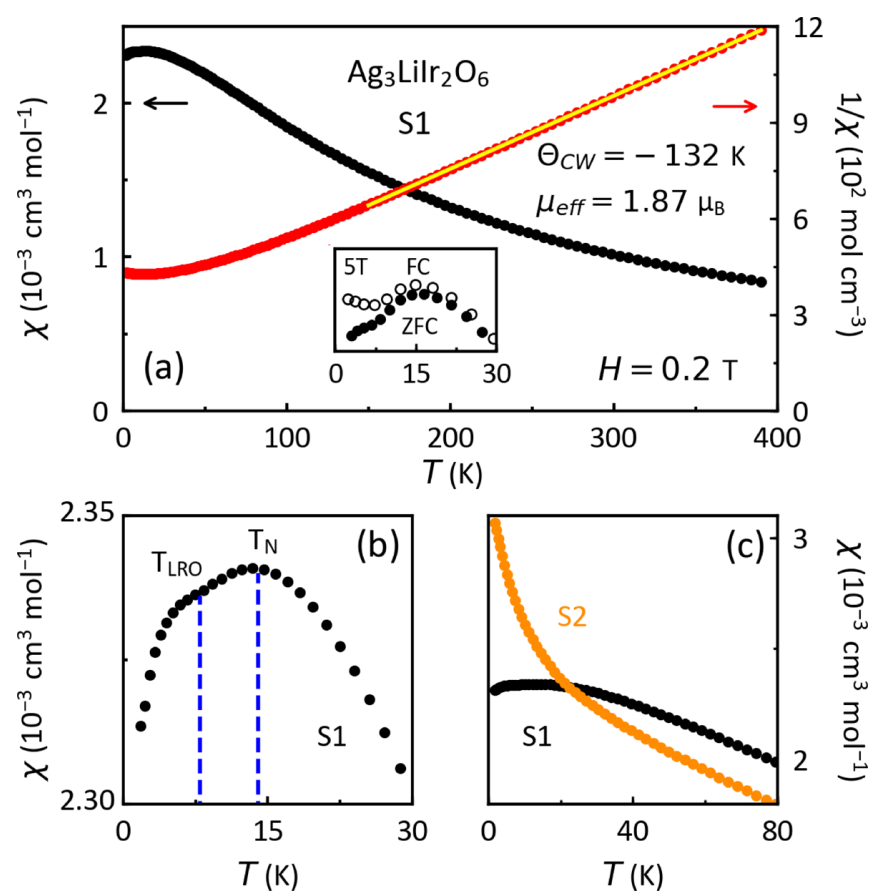

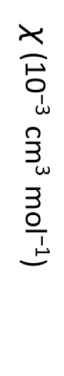

FIG. 1. (a) DC magnetic susceptibility per mole of Ir (black data) and inverse susceptibility (red data) plotted as a function of temperature in the high-quality sample, S1. The yellow line is a Curie-Weiss fit at $T>150 \mathrm{~K}$. The solid and open circles in the inset represent the zero-field-cooled (ZFC) and field-cooled (FC) curves at $H=5 \mathrm{~T}$. (b) Magnified view of the ZFC susceptibility from sample S1 showing a broad peak at $T_{N}=14 \mathrm{~K}$ and a sharper downturn at $T_{\mathrm{LRO}}=8 \mathrm{~K}$. (c) $\chi(T)$ curves are compared between the clean sample, $\mathrm{S} 1$ (black points), and the disordered sample, S2 (orange data from Ref. [14]).

the behavior of sample S2 in Fig. 1(c). The question is whether a peak is hidden under that low-temperature tail. In a similar vein, recent claims of a disordered QSL phase in $\mathrm{Cu}_{2} \mathrm{IrO}_{3}$ based on the absence of a peak in $\chi(T)$ may be questionable [18]. In fact, a small peak at $2 \mathrm{~K}$ was reported in higher-quality samples of that material and diagnosed as a signature of partial static magnetism [23].

To understand the magnetic interactions in $\mathrm{Ag}_{3} \mathrm{LiIr}_{2} \mathrm{O}_{6}$, we performed a Curie-Weiss (CW) analysis on the inverse susceptibility $1 / \chi$ in Fig. 1(a). The yellow line represents the $\mathrm{CW}$ fit that yields a $\mathrm{CW}$ temperature $\Theta_{\mathrm{CW}}=-132(1) \mathrm{K}$ and magnetic moment $\mu_{\text {eff }}=1.87(2) \mu_{B}$. The negative sign of $\Theta_{\mathrm{CW}}$ indicates AFM interactions, and its large magnitude, compared to $T_{N}$, implies magnetic frustration [24]. We extract an effective magnetic moment of $\mu_{\text {eff }}=1.87 \mu_{B}$ from the $\mathrm{CW}$ fit which is comparable to the reported values in other $\mathrm{Ki}$ taev magnets $[13,19]$ and close to the expected moment for a $J_{\text {eff }}=1 / 2$ state $\left(1.74 \mu_{B}\right)$. The values of $\mu_{\text {eff }}$ and $\Theta_{\mathrm{CW}}$ are comparable between S1 $\left(1.87 \mu_{B},-132 \mathrm{~K}\right)$ and S2 $\left(1.79 \mu_{B}\right.$, -142 K) [14].

\section{B. Heat capacity}

We measured the heat capacity $C$ of sample $\mathrm{S} 1$ to confirm the bulk AFM order in $\mathrm{Ag}_{3} \mathrm{LiIr}_{2} \mathrm{O}_{6}$. Figure 2(a) shows a broad peak in $C / T$ at $T_{N}=14 \mathrm{~K}$, consistent with the peak at $14 \mathrm{~K}$ 

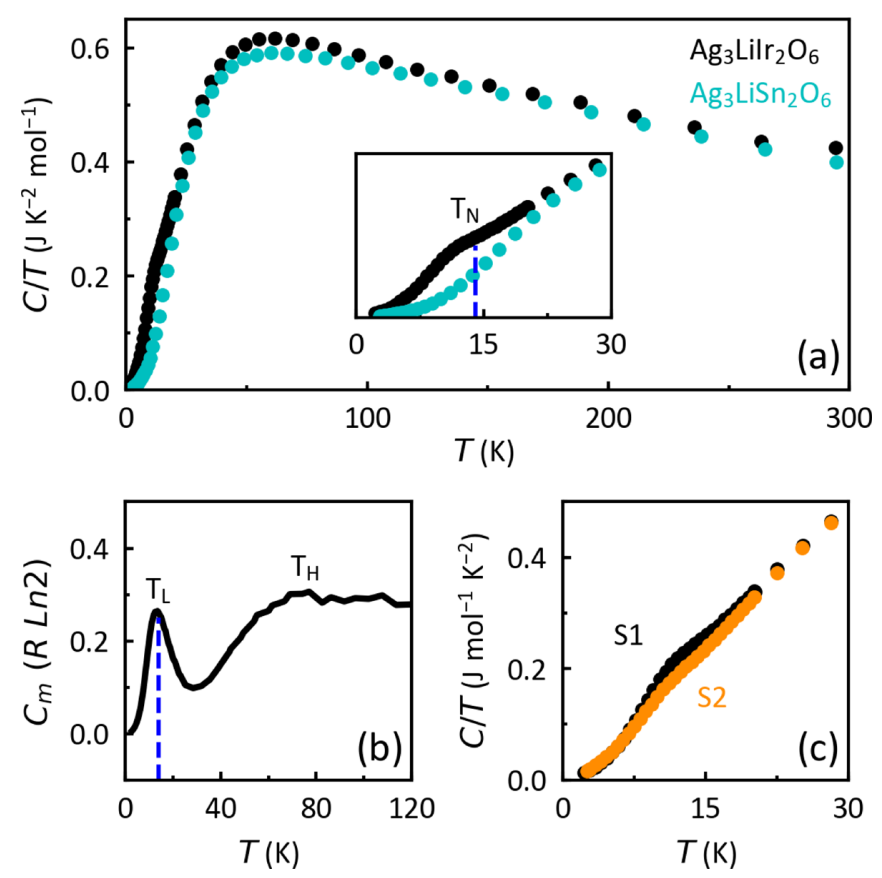

FIG. 2. (a) Heat capacity divided by temperature $(C / T)$ per mole of $\mathrm{Ir}$ or $\mathrm{Sn}$ plotted as a function of temperature in $\mathrm{Ag}_{3} \mathrm{LiIr}_{2} \mathrm{O}_{6}$ (black data) and its nonmagnetic lattice model, $\mathrm{Ag}_{3} \mathrm{LiSn}_{2} \mathrm{O}_{6}$ (turquoise data from Ref. [14]). (b) Magnetic heat capacity $C_{m}$ in units of $R \ln (2)$ as a function of temperature below $120 \mathrm{~K}$ in $\mathrm{S} 1$, where $T_{L}=T_{N}=$ $14 \mathrm{~K}$ and $T_{H}=75 \mathrm{~K}$. (c) Comparison between $C / T$ as a function of temperature below $30 \mathrm{~K}$ in the clean sample, $\mathrm{S} 1$ (black), and the disordered sample, S2 (orange). The orange curve (from Ref. [14]) is shifted by $-0.014 \mathrm{~J} \mathrm{~mol}^{-1} \mathrm{~K}^{-2}$ for clarity.

in $\chi(T)$. In the same plot, we also present the heat capacity of an isostructural compound, $\mathrm{Ag}_{3} \mathrm{LiSn}_{2} \mathrm{O}_{6}$, which serves as a nonmagnetic lattice model for $\mathrm{Ag}_{3} \mathrm{LiIr}_{2} \mathrm{O}_{6}$. The two data sets closely track each other as a function of temperature, except near 75 and $14 \mathrm{~K}$, where an additional magnetic contribution enhances the heat capacity of $\mathrm{Ag}_{3} \mathrm{LiIr}_{2} \mathrm{O}_{6}$. The magnetic heat capacity $C_{m}$ can be isolated by subtracting the $\mathrm{Ag}_{3} \mathrm{LiSn}_{2} \mathrm{O}_{6}$ data from $\mathrm{Ag}_{3} \mathrm{LiIr}_{2} \mathrm{O}_{6}$. Figure 2(b) shows $C_{m}$ in units of $R \ln (2)$ as a function of temperature where two broad peaks are resolved at a higher $T_{H}=75 \mathrm{~K}$ and a lower $T_{L}=14 \mathrm{~K}$ temperature. Such behavior has been interpreted as evidence of fractionalization of spins into Majorana fermions at $T_{H}$ followed by long-range entanglement at $T_{L}$ in $\operatorname{Ag}_{3} \mathrm{LiIr}_{2} \mathrm{O}_{6}$, $\alpha-\mathrm{Li}_{2} \mathrm{IrO}_{3}, \mathrm{Na}_{2} \mathrm{IrO}_{3}$, and $\alpha-\mathrm{RuCl}_{3}[14,19,20]$ based on a quantum Monte Carlo simulation of the Kitaev Hamiltonian [25]. We caution against this interpretation and point out that the peak at $T_{L}=T_{N}$ in $\mathrm{Ag}_{3} \mathrm{LiIr}_{2} \mathrm{O}_{6}$ is due to static magnetism instead of quantum entanglement.

We compare the $C / T$ curves between samples $\mathrm{S} 1$ (clean) and S2 (disordered) in Fig. 2(c). Whereas S2 shows a slight change in slope at $T_{N}=14 \mathrm{~K}, \mathrm{~S} 1$ reveals a peak. Notice that without the clean sample (S1), the heat capacity of S2 could have been misinterpreted as the absence of magnetic ordering. This result shows the importance of improving sample quality since without having access to $S 1$, we could not have associated the peak at $T_{L}$ with the entropy release from a long-range AFM order instead of entanglement. Similarly,

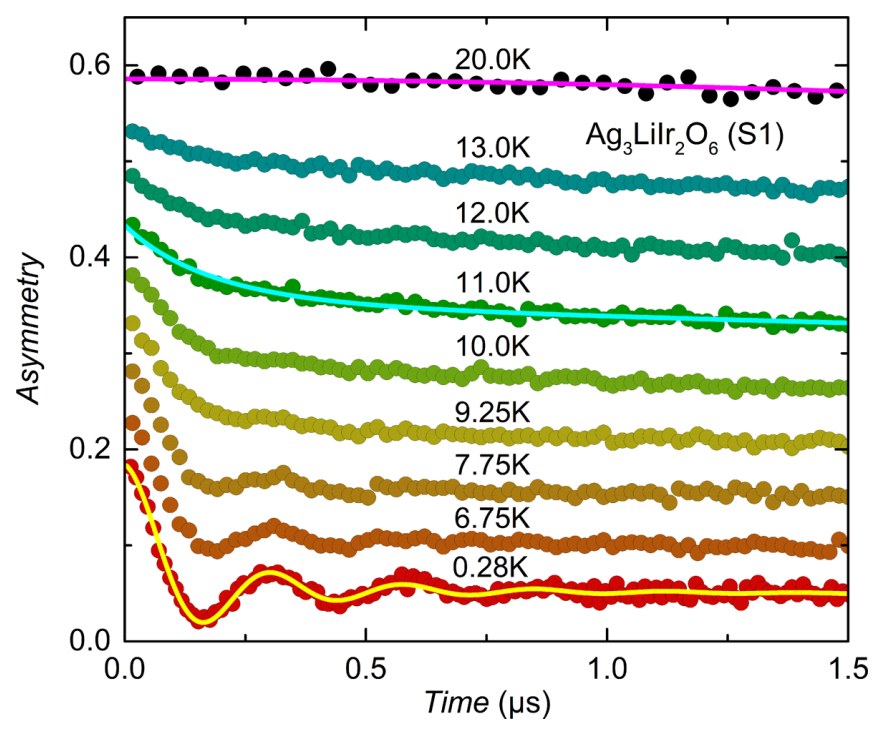

FIG. 3. Asymmetry plotted as a function of time at short timescales. The curves have been offset by equal increments from the base-temperature curve $(0.28 \mathrm{~K})$ for clarity. The magenta, cyan, and yellow solid lines are fits to Eqs. (1), (2), and (3), respectively.

the low-temperature peaks in the heat capacity of $\alpha-\mathrm{Li}_{2} \mathrm{IrO}_{3}$, $\mathrm{Na}_{2} \mathrm{IrO}_{3}$, and $\alpha-\mathrm{RuCl}_{3}$ are due to AFM ordering [14,19,20].

The above discussion does not discredit the iridate materials as candidates of a Kitaev spin liquid. Note that the peak at $T_{H}$ may, indeed, signal the onset of a fractionalization process, but the Majorana liquid develops an instability toward a gapped AFM state instead of melting into an entangled spin liquid ground state. In $\alpha-\mathrm{RuCl}_{3}$, this instability is removed by applying a 7-T magnetic field parallel to the honeycomb planes [26]. A similar effect may be observed in $\mathrm{Ag}_{3} \mathrm{LiIr}_{2} \mathrm{O}_{6}$ once single crystals are available.

\section{Muon spin relaxation}

In positive muon spin relaxation $\left(\mu^{+} \mathrm{SR}\right)$, spin-polarized positive muons are injected into a sample and in less than 1 ps come to rest at a preferred crystallographic interstitial site (or sites). The muon spin polarization then evolves with time in the local magnetic field, yielding information about the magnitude and orientation of the local field relative to the initial spin direction. After tens of millions of decay events, a time histogram can be used to extract the asymmetry, which is proportional to the time dependence of the projection of the muon spin along the detector direction [27]. The asymmetry contains information about the local field's temporal and spatial variation.

We plot the asymmetry as a function of time in sample $\mathrm{S} 1$ in Fig. 3 at nine representative temperatures from 0.28 to $20 \mathrm{~K}$ at zero field. For temperatures greater than or equal to $20 \mathrm{~K}$, the $\mathrm{Ir}^{4+}$ moments are fluctuating too rapidly, and they have no effect on the muon. Therefore, the depolarization is dominated by randomly oriented quasistatic nuclear moments. The temperature-dependent asymmetry is well described by a Gaussian Kubo-Toyabe function,

$$
A_{\mathrm{KT}}(t)=A_{0}\left[\frac{1}{3}+\frac{2}{3}\left(1-\sigma^{2} t^{2}\right) \exp \left(-\frac{1}{2} \sigma^{2} t^{2}\right)\right],
$$


where $A_{0}=0.174$ is the initial asymmetry for GPS in the spin-rotated mode and the parameter $\sigma=0.150 \mathrm{MHz}$ is proportional to the second moment of the field distribution experienced by the muon ensemble. The magenta line in Fig. 3 is a fit to Eq. (1) at $20 \mathrm{~K}$. We found a constant value for $\sigma$ between 200 and $20 \mathrm{~K}$, indicating that the muon is not diffusing in this temperature range. The data below $20 \mathrm{~K}$ can be explained in three regions of interest.

Region 1. For $20 \mathrm{~K}>T>T_{N}$, depolarization is dominated by the nuclear moments. The electronic moments are slowing down and begin contributing to muon depolarization.

Region 2. For the range $T_{N}>T>T_{\mathrm{LRO}}$, depolarization is dominated by the electronic moments. Short-range correlations are manifested in the onset of a fast relaxation component in addition to a slow exponential depolarization due to fluctuations. To characterize the crossover in this temperature range, we use a phenomenological depolarization function,

$$
A(t)=A_{0}\left[\alpha_{F} \exp \left(-\left(\lambda_{F} t\right)^{\beta}\right)+\left(1-\alpha_{F}\right) \exp \left(-\lambda_{S} t\right)\right],
$$

where $A_{0}=0.185$ is the initial asymmetry in the Dolly spectrometer in spin-rotated mode. The first term in the brackets is related to the fast decay with rate $\lambda_{F}$ best described by a stretched exponential with exponent $\beta$ and attributed to spin freezing. The second term is a slow exponential decay at rate $\lambda_{S}$ attributed to a fluctuating contribution. The fit parameters $\lambda_{F}, \beta$, and $\lambda_{S}$ in sample S1 vary from 10.1(6) $\mu \mathrm{s}^{-1}, 0.85(6)$, and $0.211(2) \mu \mathrm{s}^{-1}$ at $13 \mathrm{~K}$, respectively, to $11.0(1) \mu \mathrm{s}^{-1}$, $1.75(5)$, and $0.285(8) \mu \mathrm{s}^{-1}$ at $8 \mathrm{~K}$.

The cyan line in Fig. 3 is a representative fit to Eq. (2) at $11 \mathrm{~K}$. From such fits, we extract the fraction of fast decay $\alpha_{F}$, which we take as a metric for the onset of static magnetism. The temperature dependence of $\alpha_{F}$ is plotted in Fig. 4(a), and it vanishes near $T_{N}=14 \mathrm{~K}$.

We compare the polarization (normalized asymmetry) at $10 \mathrm{~K}$ between samples S1 and S2 in Fig. 4(b). At this temperature $\left(T_{N}>T>T_{\mathrm{LRO}}\right)$, neither $\mathrm{S} 1$ nor $\mathrm{S} 2$ shows oscillations; however, the fast decay below $1 \mu \mathrm{s}$ is visibly faster in $\mathrm{S} 1$. Note that the long-time tail of polarization converges to the same value in both samples, indicating weak dynamics. We conclude that the same magnetic ordering starts below $T_{N}$ in both samples, but the short-range correlations are stronger in $\mathrm{S} 1$, evidenced by larger $\lambda_{F}$ than in S2.

Region 3. At $T<T_{\mathrm{LRO}}$, clear oscillations appear in the depolarization curves of S1 (Fig. 3), indicating a long-range magnetic order. The depolarization curves are well described by the function

$$
\begin{aligned}
A_{\mathrm{LRO}}(t)= & A_{0}\left[\alpha_{\mathrm{LRO}} \exp (-\Lambda t) J_{0}\left(\gamma_{\mu} B_{\mathrm{max}} t+\phi\right)\right. \\
& \left.+\left(1-\alpha_{\mathrm{LRO}}\right) \exp (-\lambda t)\right] .
\end{aligned}
$$

Again, the initial asymmetry is $A_{0}=0.185$ in the Dolly spectrometer. Here $J_{0}$ is the zeroth-order Bessel function, and the muon gyromagnetic ratio is $\gamma_{\mu}=2 \pi(135.5 \mathrm{MHz} / \mathrm{T})$. The yellow line in Fig. 3 is a fit to the Bessel function at $0.28 \mathrm{~K}$. A Bessel oscillatory behavior is typically associated with incommensurate magnetic ordering [27], where the muon experiences ordered fields ranging from zero to $B_{\max }$. We extract the $B_{\max }$ value from such a fit at each temperature below $T_{\text {LRO }}$ and plot it in Fig. 4(a) as red squares. Such an analysis
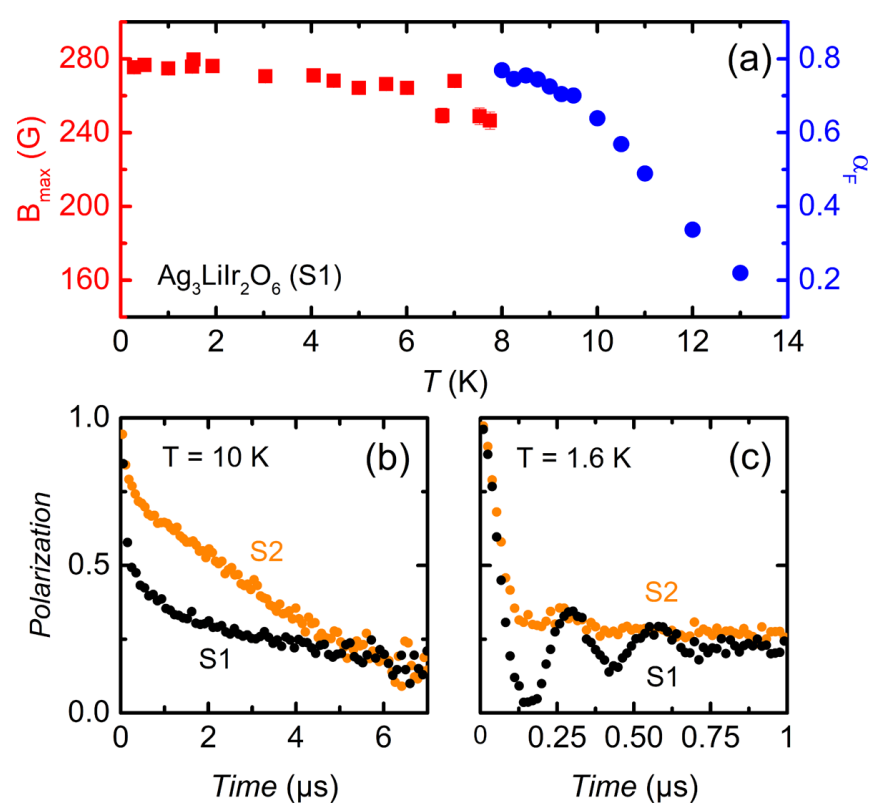

FIG. 4. (a) The blue circles represent $\alpha_{F}$ values from fits to Eq. (2), and the red squares represent $B_{\max }$ values from fits to Eq. (3) in the clean sample, S1. Static magnetism starts at $T_{N}=14 \mathrm{~K}$, and $\mu \mathrm{SR}$ oscillations start at $T_{L R O}=8 \mathrm{~K}$. (b) Muon polarization ( $P=A / A_{0}$, where $A_{0}$ is the initial asymmetry) as a function of time in $\mathrm{S} 1$ and $\mathrm{S} 2$ at $10 \mathrm{~K}\left(T_{N}>T>T_{L R O}\right)$. (c) Polarization curves below $1 \mu \mathrm{s}$ in $\mathrm{S} 1$ and $\mathrm{S} 2$ at $1.6 \mathrm{~K}\left(T<T_{L R O}\right)$. The oscillations are barely discernible in the disordered sample $\mathrm{S} 2$, although the initial depolarization is comparable between $\mathrm{S} 1$ and $\mathrm{S} 2$.

would be impossible for the disordered sample, $\mathrm{S} 2$, as can be seen from the comparison in Fig. 4(c). The oscillations are barely visible in S2; thus, a fit to Eq. (3) would not work. Two additional observations in Fig. 4(c) are worth noting. First, at an extremely short timescale (less than $0.1 \mu \mathrm{s}$ ), the fast depolarization is identical in both samples. Second, the long-time depolarization tail $(t>0.8 \mu \mathrm{s})$ converges between the two samples. From these observations, we conclude that a similar incommensurate order exists in the ground state of both samples, but with a longer correlation length in sample $\mathrm{S} 1$ than in $\mathrm{S} 2$ because it has less disorder.

At the base temperature $T=0.28 \mathrm{~K}$, the fit to Eq. (3) yields $\alpha_{\mathrm{LRO}}=0.741(2), B_{\max }=269(1) \mathrm{G}, \phi=-0.9(6)^{\circ}$, $\Lambda=2.8(1) \mu \mathrm{s}^{-1}$, and $\lambda=0.052(4) \mu \mathrm{s}^{-1}$. The value of $\alpha_{\mathrm{LRO}}$ is close to the value of $2 / 3$ expected from a polycrystalline sample exhibiting long-range magnetic order. The value of $B_{\max }$ is confirmed from a longitudinal field (LF) experiment in Appendix C. The damping rate $\lambda$ is associated with those muons whose initial polarization lies along the local magnetic field and are depolarized by transverse magnetic fluctuations. The rate $\Lambda$ contains contributions from both static magnetic disorder and magnetic fluctuations. Since $\Lambda \gg \lambda$, disorder is the dominant contribution.

\section{Transmission electron microscopy}

So far, we have presented the magnetic behavior of $\mathrm{Ag}_{3} \mathrm{LiIr}_{2} \mathrm{O}_{6}$ in the clean (S1) and disordered (S2) limits using both bulk and local probes. Here we characterize the structural 

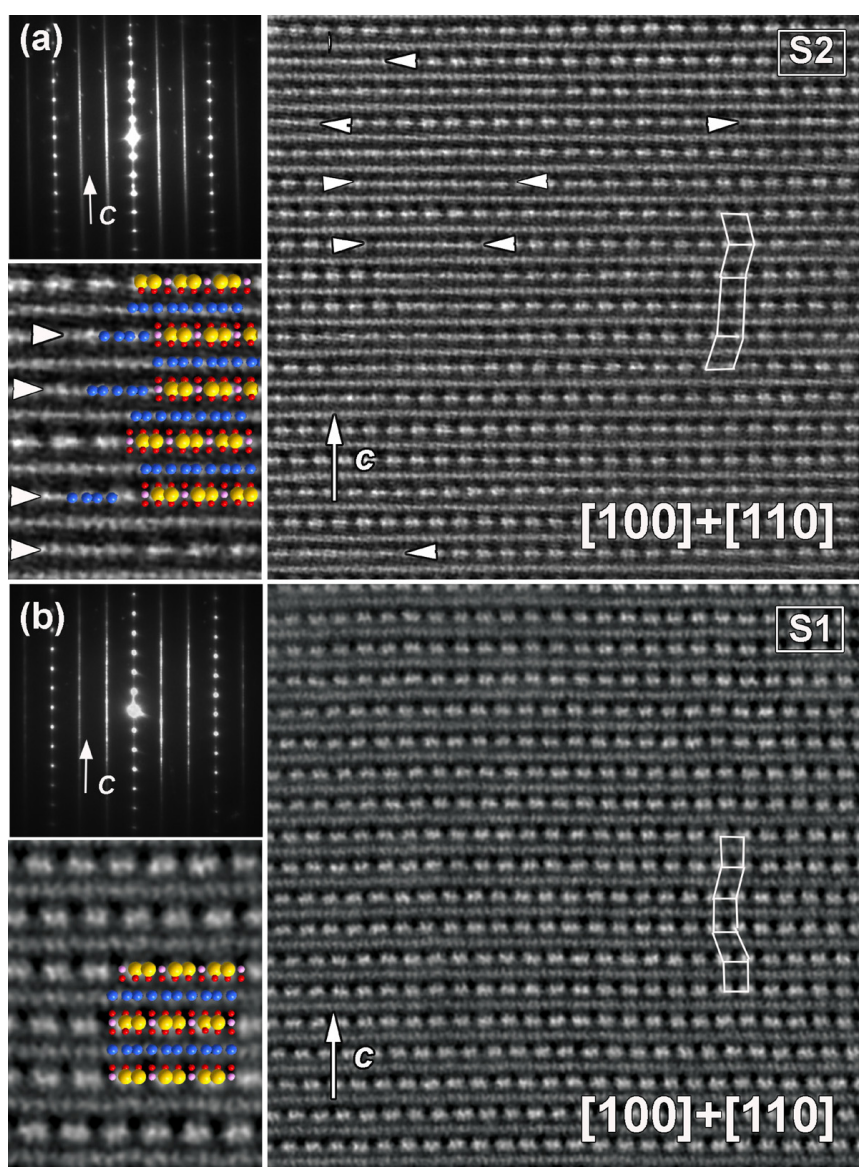

FIG. 5. (a) Electron diffraction (top inset) and HAADF-STEM image from the disordered sample, S2. A structural model is overlaid on the magnified image in the bottom inset with blue, yellow, pink, and red spheres for the $\mathrm{Ag}, \mathrm{Ir}, \mathrm{Li}$, and $\mathrm{O}$ atoms, respectively. The arrows indicate where $\mathrm{Ag}$ atoms replace Ir atoms within the honeycomb layers. (b) Similar images from the clean sample, S1, where Ag inclusion is absent.

disorder in the material using high-resolution HAADF-STEM images from both samples S1 and S2 in Fig. 5. The characteristic feature of each honeycomb layer in Figs. 5(a) and 5 (b) is a repeating pattern of a pair of Ir atoms (large bright spots) separated by a $\mathrm{Li}$ atom (not visible). This pattern is interrupted in sample S2 by rows of unwanted Ag atoms (smaller bright spots), as indicated by the arrows in Fig. 5(a). Note that silver inclusions take the form of extended defects (rows of Ag atoms) instead of local defects (singular intersite disorder). The distinction between local and extended defects is important, especially in theoretical modeling of disordered Kitaev magnets [28].

In the inset in Fig. 5(a), a crystallographic model is overlaid on the magnified image to identify the $\mathrm{Ag}$, $\mathrm{Ir}, \mathrm{Li}$, and $\mathrm{O}$ atoms as blue, yellow, pink, and red spheres, respectively (only the $\mathrm{Ag}$ and Ir atoms are clearly visible). The arrows indicate where the unwanted $\mathrm{Ag}$ atoms (blue) are inserted within the Ir layer (yellow). In contrast, the HAADF-STEM image from the clean sample $\mathrm{S} 1$ in Fig. 5(b) shows pristine honeycomb layers free from silver inclusions.

We present the ED patterns for S1 and S2 in the top insets of Figs. 5(a) and 5(b). The streaking in ED patterns is due to the stacking faults in the form of angular twist between the adjacent layers, as shown in other honeycomb materials [29]. Upon careful inspection, the ED pattern of sample S1 reveals less streaking than $\mathrm{S} 2$. This is consistent with the synthesis of sample $\mathrm{S} 1$ from a precursor $\alpha-\mathrm{Li}_{2} \mathrm{IrO}_{3}$ with fewer stacking faults, as explained in Appendix A (Fig. 6). We show in Appendix D (Fig. 9) that $\mathrm{Ag}_{3} \mathrm{LiIr}_{2} \mathrm{O}_{6}$ has more stacking faults than its parent compounds $\alpha-\mathrm{Li}_{2} \mathrm{IrO}_{3}$. It is likely that in the absence of such stacking faults, the initial spin freezing at $T_{N}$ could turn into a long-range order, i.e., $T_{N}=T_{\mathrm{LRO}}[30]$.

\section{CONCLUSION}

By improving the sample quality, we have revealed signatures of a long-range incommensurate order in $\mathrm{Ag}_{3} \mathrm{LiIr}_{2} \mathrm{O}_{6}$. A broad peak in the magnetic susceptibility and heat capacity at $T_{N}=14 \mathrm{~K}$ marks the onset of magnetic ordering. Such a peak is absent in the disordered sample, which hinders the recognition of a long-range order in $\mathrm{Ag}_{3} \mathrm{LiIr}_{2} \mathrm{O}_{6}$. In $\mu \mathrm{SR}$, a fast decay of muon depolarization below $T_{N}$ shows the onset of short-range order, and the appearance of oscillations below $T_{\mathrm{LRO}}$ confirms the long-range order. The oscillation patterns at low temperatures fit to a Bessel function, consistent with incommensurate ordering. An incommensurate spiral order has been confirmed in $\alpha-\mathrm{Li}_{2} \mathrm{IrO}_{3}$ from both $\mu \mathrm{SR}$ and neutron scattering [31]. Our HAADF-STEM images confirm a moderate level of extended defects (silver inclusion) in the disordered $\mathrm{Ag}_{3} \mathrm{LiIr}_{2} \mathrm{O}_{6}$ sample made from a lower-quality $\alpha-\mathrm{Li}_{2} \mathrm{IrO}_{3}$. In the disordered sample, the $\mathrm{Ag}$ atoms enter the honeycomb layer and disrupt the long-range magnetic order. This effect must be distinguished from the lack of magnetic ordering due to long-range entanglement in a quantum spin liquid.

\section{ACKNOWLEDGMENTS}

We thank R. Valenti, N. B. Perkins, and J. Knolle for fruitful discussions and H. Luetkens, T. Shiroka, and C. Baines for their technical assistance with the $\mu \mathrm{SR}$ experiments. The work at Boston College was supported by the National Science Foundation under Award No. DMR-1708929. This work is based on experiments performed at the Swiss Muon Source $\mathrm{S} \mu \mathrm{S}$ at the Paul Scherrer Institute, Villigen, Switzerland, and at the ISIS Pulsed Muon Source, which is supported by the U.K. Science and Technology Facilities Council.

F.B. and E.M.K. contributed equally to this work.

\section{APPENDIX A: SYNTHESIS DETAILS}

The important difference between the two $\mathrm{Ag}_{3} \mathrm{LiIr}_{2} \mathrm{O}_{6}$ samples, $\mathrm{S} 1$ and $\mathrm{S} 2$, is in the $\alpha-\mathrm{Li}_{2} \mathrm{IrO}_{3}$ precursor used in their synthesis. Figure 6(a) compares the x-ray patterns between two $\alpha-\mathrm{Li}_{2} \mathrm{IrO}_{3}$ precursors, shown in black and red, used for the synthesis of samples S1 (clean) and S2 (disordered), respectively. The region between $19^{\circ}$ and $24^{\circ}$ gives information about the quality of honeycomb ordering in $\alpha-\mathrm{Li}_{2} \mathrm{IrO}_{3}$ [Fig. 6(a), left inset]. The black x-ray pattern with sharp and well-separated peaks indicates better honeycomb ordering and fewer stacking faults than the red x-ray pattern. A similar level of disorder carries over to the $\mathrm{Ag}_{3} \mathrm{LiIr}_{2} \mathrm{O}_{6}$ produced from these precursors. We also reveal the effect of disorder on the 

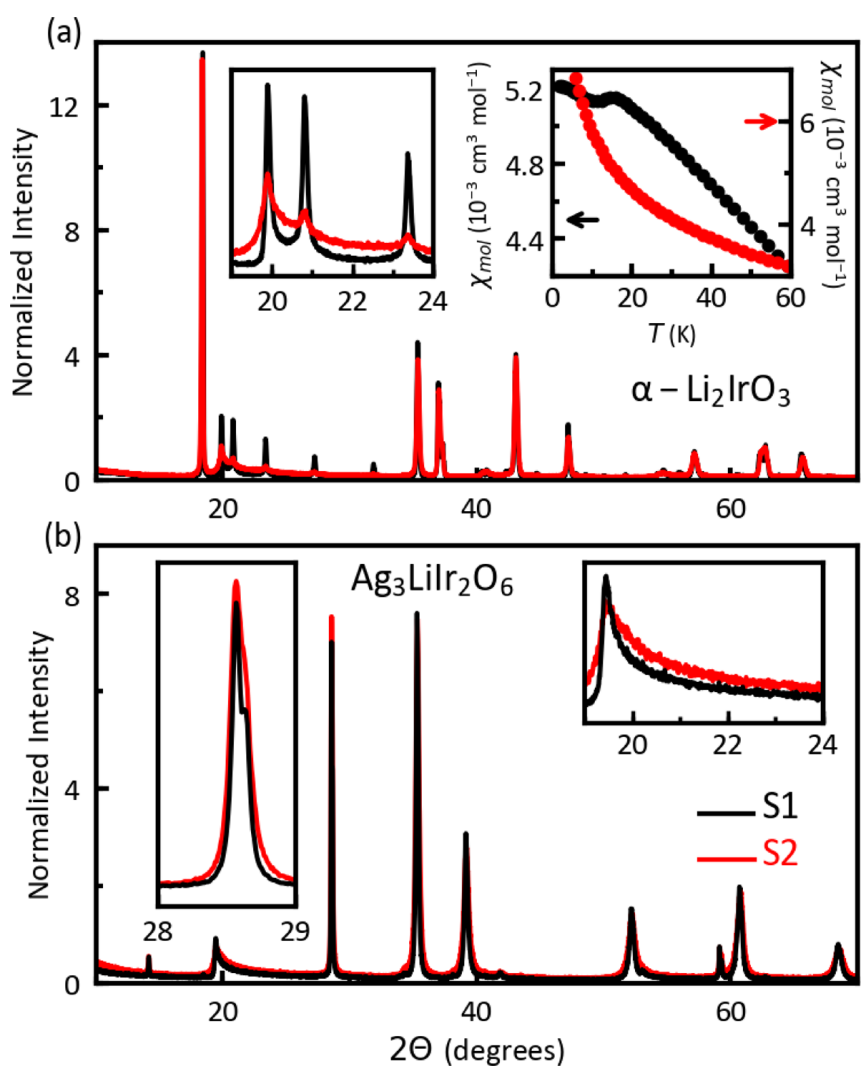

FIG. 6. (a) X-ray patterns of two $\alpha-\mathrm{Li}_{2} \mathrm{IrO}_{3}$ precursors used in the synthesis of clean (black) and disordered (red) $\mathrm{Ag}_{3} \mathrm{LiIr}_{2} \mathrm{O}_{6}$. The region of honeycomb peaks is magnified in the left inset. The temperature dependence of the DC magnetic susceptibility in the two $\alpha-\mathrm{Li}_{2} \mathrm{IrO}_{3}$ precursors is presented in the right inset. (b) X-ray patterns of two $\mathrm{Ag}_{3} \mathrm{LiIr}_{2} \mathrm{O}_{6}$ samples, S1 (black) and S2 (red, from Ref. [14]). The peaks at $28.5^{\circ}$ in the two $\mathrm{Ag}_{3} \operatorname{LiIr}_{2} \mathrm{O}_{6}$ samples are compared in the left inset. The region of honeycomb peaks is magnified in the right inset.

magnetic behavior of $\alpha-\mathrm{Li}_{2} \mathrm{IrO}_{3}$ by plotting DC susceptibility of both $\alpha-\mathrm{Li}_{2} \mathrm{IrO}_{3}$ samples as a function of temperature below $60 \mathrm{~K}$ in the right inset in Fig. 6(a). The red curve does not show any signs of magnetic ordering, while the black curve shows a peak at the AFM transition at $15 \mathrm{~K}$.

Figure 6(b) shows the difference between the X-ray patterns of $\mathrm{Ag}_{3} \mathrm{LiIr}_{2} \mathrm{O}_{6}$ samples $\mathrm{S} 1$ (black) and $\mathrm{S} 2$ (red). The main differences between the two samples are the intensity of the peak at $28.5^{\circ}$ (left inset) and the sharpness in the asymmetric honeycomb peaks (right inset). S1 has sharper asymmetric honeycomb peaks and a shorter peak at $28.5^{\circ}$, which is similar to a prior report [15]. Whereas we have used $\mathrm{AgNO}_{3}$ for the silver-exchange reaction, the authors of Ref. [15] used a mixture of $\mathrm{AgNO}_{3} / \mathrm{KNO}_{3}$ for the reaction. In S2, the honeycomb peaks are broader, and the intensities of the two peaks at $28.5^{\circ}$ and $35.3^{\circ}$ are nearly the same.

\section{APPENDIX B: SPLITTING BETWEEN ZFC AND FC DATA}

In Fig. 7, we show the splitting between ZFC and FC susceptibility at several fields. Note that the splitting persists to high fields, confirming a static spin freezing [32,33] at $T_{N}$, as noted in the main text.
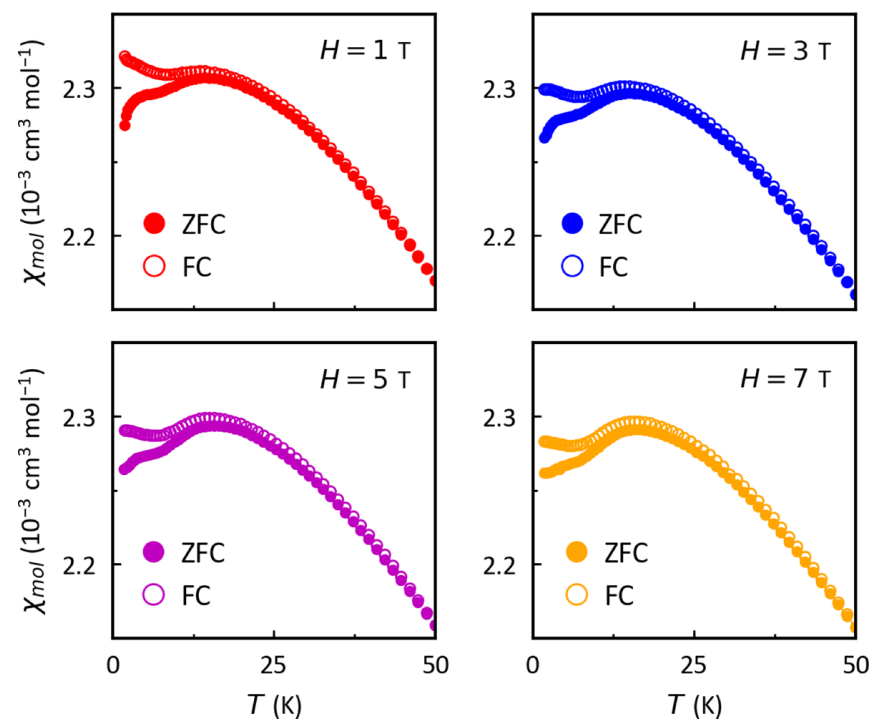

FIG. 7. The splitting between ZFC (solid circles) and FC (open circles) susceptibility curves at $1,3,5$, and $7 \mathrm{~T}$.

\section{APPENDIX C: $\mu$ SR DATA UNDER LONGITUDINAL FIELD}

In the main text, we derived $B_{\max }=269 \mathrm{G}$ in sample $\mathrm{S} 1$ at $0.28 \mathrm{~K}$ by fitting the zero-field (ZF) $\mu \mathrm{SR}$ data to a Bessel function [Eq. (3)]. As a consistency check, here we estimate the internal field $B_{\text {int }}$ by analyzing the longitudinal field (LF) scans at $0.28 \mathrm{~K}$, as shown in Fig. 8(a). The initial polarization
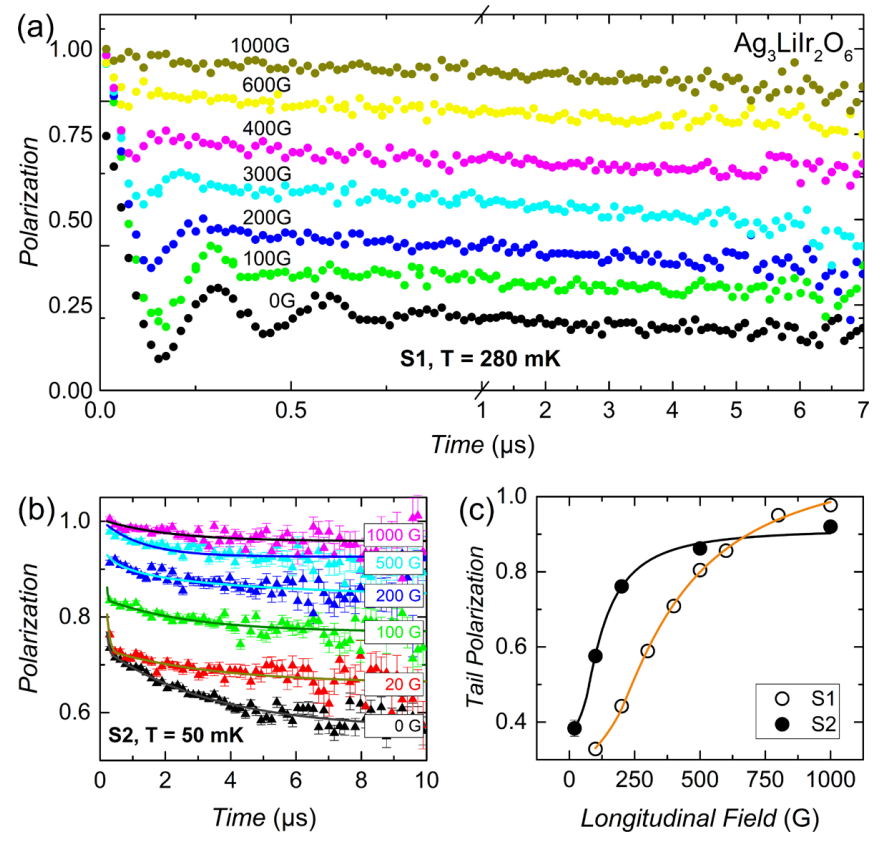

FIG. 8. (a) Polarization scans in the clean sample, S1, at $0.28 \mathrm{~K}$ under different longitudinal fields (LFs) from 0 to $1000 \mathrm{G}$. The time axis is expanded for $t<1 \mu$ s to reveal the oscillations. (b) Polarization scans in the disordered sample, S2, at $0.05 \mathrm{~K}$ under LFs from 0 to $1000 \mathrm{G}$. The data in (a) and (b) were collected at the PSI and ISIS facilities, respectively. (c) By analyzing the recovery of the initial asymmetry with increasing field, we estimate $B_{\text {int }}=263 \mathrm{G}$ in S1 and $113 \mathrm{G}$ in $\mathrm{S} 2$. Solid lines are guides to the eye. 
is fully recovered by $1000 \mathrm{G}$, so the internal field $B_{\text {int }}$ must be much smaller than this value. A detailed analysis [34] shows that the midpoint of the polarization recovery occurs at a field value close to $B / B_{\text {int }}=4 / 3$. Figure 8 (c) shows that the midpoint of recovery in $\mathrm{S} 1$ is at $350 \mathrm{G}$, yielding an internal field $B_{\text {int }}=263 \mathrm{G}$, in good agreement with $B_{\max }=269 \mathrm{G}$ obtained from our Bessel function fit to Eq. (3). We have also collected LF scans from the disordered sample (S2) at $0.05 \mathrm{~K}$, as shown in Fig. 8(b). The midpoint of recovery in $\mathrm{S} 2$ occurs at $150 \mathrm{G}$ in Fig. 8(c), yielding an internal field $B_{\text {int }}=113 \mathrm{G}$, which is smaller than in sample S1. A smaller internal field may result from a range of muon stopping sites in the disordered sample. Since $\mu$ SR is a local probe, we do not expect a major change in the local field near $\mathrm{Ir}^{4+}$ sites below $T_{N}$, but it is likely that muons probe a range of stopping sites with slightly different chemical environments due to various levels of $\mathrm{Ag}$ inclusion across the sample. This explains the slow depolarization of muons inside S2 at $10 \mathrm{~K}$ in Fig. 4, and the different polarization recoveries between $\mathrm{S} 2$ and $\mathrm{S} 1$ in Fig. 8(c). As noted in the main text, it is not possible to fit the ZF data in sample S2 to a Bessel function [Eq. (3)] because the oscillations are not discernible in the disordered sample. Thus, the LF analysis is the only way of estimating the local internal field in $\mathrm{S} 2$.

\section{APPENDIX D: TEM ANALYSIS OF THE STACKING FAULTS}

Our discussion of the structural disorder in the main text focused on the $\mathrm{Ag}$ inclusion within the honeycomb layers of $\mathrm{Ag}_{3} \mathrm{LiIr}_{2} \mathrm{O}_{6}$ (Fig. 5). Here we point out that both the clean (S1) and disordered (S2) samples of $\mathrm{Ag}_{3} \mathrm{LiIr}_{2} \mathrm{O}_{6}$ also suffer from stacking faults, similar to other layered honeycomb materials such as $\mathrm{Cu}_{3} \mathrm{LiSn}_{2} \mathrm{O}_{6}$ [29]. Figure 9 compares HAADF-TEM images between a clean sample of $\alpha-\mathrm{Li}_{2} \mathrm{IrO}_{3}$ and a clean sample of $\mathrm{Ag}_{3} \mathrm{LiIr}_{2} \mathrm{O}_{6}(\mathrm{~S} 1)$. There is no intersite disorder in either image, but $\mathrm{Ag}_{3} \mathrm{LiIr}_{2} \mathrm{O}_{6}$ exhibits many more stacking faults than its parent compound, $\alpha-\mathrm{Li}_{2} \mathrm{IrO}_{3}$. It was demonstrated in a prior study of $\mathrm{Cu}_{3} \mathrm{LiSn}_{2} \mathrm{O}_{6}$ that the stacking faults result from a twisting between the adjacent honeycomb layers due to the weak $\mathrm{O}-\mathrm{Cu}-\mathrm{O}$ dumbbell bonds between the layers [29]. A similar mechanism is at work in $\mathrm{Ag}_{3} \mathrm{LiIr}_{2} \mathrm{O}_{6}$, where the weak $\mathrm{O}-\mathrm{Ag}-\mathrm{O}$ dumbbell bonds lead to the twisting

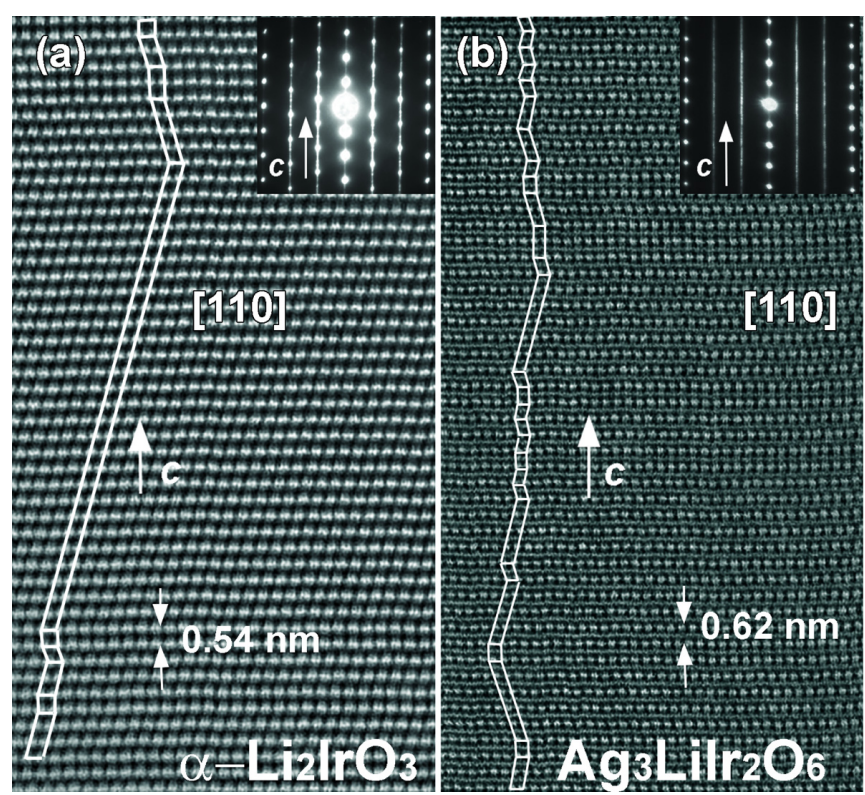

FIG. 9. HAADF-TEM images from (a) $\alpha-\mathrm{Li}_{2} \mathrm{IrO}_{3}$ and (b) $\mathrm{Ag}_{3} \mathrm{LiIr}_{2} \mathrm{O}_{6}$ (S1). A clean sample is used for each material. The magnetization and $\mathrm{x}$-ray data for the $\alpha-\mathrm{Li}_{2} \mathrm{IrO}_{3}$ sample are presented in Fig. 6 (black data). The magnetization data for the $\mathrm{Ag}_{3} \operatorname{LiIr}_{2} \mathrm{O}_{6}$ are presented in the main text (sample S1). The images show an abundance of stacking faults in $\mathrm{Ag}_{3} \mathrm{LiIr}_{2} \mathrm{O}_{6}$, unlike $\alpha-\mathrm{Li}_{2} \mathrm{IrO}_{3}$, due to the weaker interlayer coupling in the former. The ED patterns are presented as insets and reveal less streaking in $\alpha-\mathrm{Li}_{2} \mathrm{IrO}_{3}$ due to fewer stacking faults compared to $\mathrm{Ag}_{3} \mathrm{LiIr}_{2} \mathrm{O}_{6}$.

between the layers and produce the zigzag stacking pattern observed in Fig. 9(b). Despite the considerable number of stacking faults in sample S1 [Fig. 9(b)], it still shows clear signatures of long-range order, as explained in the main text. In fact, the incommensurate order is similar between $\alpha-\mathrm{Li}_{2} \mathrm{IrO}_{3}$ and $\mathrm{Ag}_{3} \mathrm{LiIr}_{2} \mathrm{O}_{6}$ based on our $\mu \mathrm{SR}$ data and the published results in Ref. [31]. Thus, we conclude that the magnetic interactions within the honeycomb layers are not affected by the interlayer bonds; however, they are disrupted by the extended defects in the form of silver inclusion within the honeycomb layers.
[1] C. Broholm, R. J. Cava, S. A. Kivelson, D. G. Nocera, M. R. Norman, and T. Senthil, Science 367, eaay0668 (2020).

[2] J. Knolle and R. Moessner, Annu. Rev. Condens. Matter Phys. 10, 451 (2019).

[3] L. Savary and L. Balents, Rep. Prog. Phys. 80, 016502 (2016).

[4] A. Kitaev, Ann. Phys.(NY), 321, 2 (2006).

[5] G. Jackeli and G. Khaliullin, Phys. Rev. Lett. 102, 017205 (2009).

[6] Y. Singh, S. Manni, J. Reuther, T. Berlijn, R. Thomale, W. $\mathrm{Ku}, \mathrm{S}$. Trebst, and P. Gegenwart, Phys. Rev. Lett. 108, 127203 (2012)

[7] H. Takagi, T. Takayama, G. Jackeli, G. Khaliullin, and S. E. Nagler, Nat. Rev. Phys. 1, 264 (2019).
[8] K. W. Plumb, J. P. Clancy, L. J. Sandilands, V. V. Shankar, Y. F. Hu, K. S. Burch, H.-Y. Kee, and Y.-J. Kim, Phys. Rev. B 90, 041112(R) (2014).

[9] J. Nasu, J. Knolle, D. L. Kovrizhin, Y. Motome, and R. Moessner, Nat. Phys. 12, 912 (2016).

[10] Y. Wang, G. B. Osterhoudt, Y. Tian, P. Lampen-Kelley, A. Banerjee, T. Goldstein, J. Yan, J. Knolle, H. Ji, R. J. Cava, J. Nasu, Y. Motome, S. E. Nagler, D. Mandrus, and K. S. Burch, npj Quantum Mater. 5, 14 (2020).

[11] K. Kitagawa, T. Takayama, Y. Matsumoto, A. Kato, R. Takano, Y. Kishimoto, S. Bette, R. Dinnebier, G. Jackeli, and H. Takagi, Nature (London) 554, 341 (2018).

[12] J. H. Roudebush, K. A. Ross, and R. J. Cava, Dalton Trans. 45, 8783 (2016). 
[13] M. Abramchuk, C. Ozsoy-Keskinbora, J. W. Krizan, K. R. Metz, D. C. Bell, and F. Tafti, J. Am. Chem. Soc. 139, 15371 (2017).

[14] F. Bahrami, W. Lafargue-Dit-Hauret, O. I. Lebedev, R. Movshovich, H.-Y. Yang, D. Broido, X. Rocquefelte, and F. Tafti, Phys. Rev. Lett. 123, 237203 (2019).

[15] V. Todorova, A. Leineweber, L. Kienle, V. Duppel, and M. Jansen, J. Solid State Chem. 184, 1112 (2011).

[16] I. Kimchi, J. P. Sheckelton, T. M. McQueen, and P. A. Lee, Nat. Commun. 9, 4367 (2018).

[17] J. Knolle, R. Moessner, and N. B. Perkins, Phys. Rev. Lett. 122, 047202 (2019).

[18] Y. S. Choi, C. H. Lee, S. Lee, S. Yoon, W.-J. Lee, J. Park, A. Ali, Y. Singh, J.-C. Orain, G. Kim, J.-S. Rhyee, W.-T. Chen, F. Chou, and K.-Y. Choi, Phys. Rev. Lett. 122, 167202 (2019).

[19] K. Mehlawat, A. Thamizhavel, and Y. Singh, Phys. Rev. B 95, 144406 (2017).

[20] S. Widmann, V. Tsurkan, D. A. Prishchenko, V. G. Mazurenko, A. A. Tsirlin, and A. Loidl, Phys. Rev. B 99, 094415 (2019).

[21] A. Suter and B. M. Wojek, Phys. Procedia 30, 69 (2012).

[22] S. Bette, T. Takayama, K. Kitagawa, R. Takano, H. Takagi, and R. E. Dinnebier, Dalton Trans. 46, 15216 (2017).

[23] E. M. Kenney, C. U. Segre, W. Lafargue-Dit-Hauret, O. I. Lebedev, M. Abramchuk, A. Berlie, S. P. Cottrell, G. Simutis, F. Bahrami, N. E. Mordvinova, G. Fabbris, J. L. McChesney, D. Haskel, X. Rocquefelte, M. J. Graf, and F. Tafti, Phys. Rev. B 100, 094418 (2019).
[24] A. P. Ramirez, Annu. Rev. Mater. Sci. 24, 453 (1994).

[25] J. Nasu, M. Udagawa, and Y. Motome, Phys. Rev. B 92, 115122 (2015).

[26] A. Banerjee, P. Lampen-Kelley, J. Knolle, C. Balz, A. A. Aczel, B. Winn, Y. Liu, D. Pajerowski, J. Yan, C. A. Bridges, A. T. Savici, B. C. Chakoumakos, M. D. Lumsden, D. A. Tennant, R. Moessner, D. G. Mandrus, and S. E. Nagler, npj Quantum Mater. 3, 1 (2018).

[27] A. Yaouanc and P. Dalmas de Réotier, Muon Spin Rotation, Relaxation, and Resonance: Applications to Condensed Matter, International Series of Monographs on Physics (Oxford University Press, Oxford, 2011).

[28] W.-H. Kao, J. Knolle, G. B. Halász, R. Moessner, and N. B. Perkins, Phys. Rev. X 11, 011034 (2021).

[29] M. Abramchuk, O. I. Lebedev, O. Hellman, F. Bahrami, N. E. Mordvinova, J. W. Krizan, K. R. Metz, D. Broido, and F. Tafti, Inorg. Chem. 57, 12709 (2018).

[30] I. Rousochatzakis, S. Kourtis, J. Knolle, R. Moessner, and N. B. Perkins, Phys. Rev. B 100, 045117 (2019).

[31] S. Choi, S. Manni, J. Singleton, C. V. Topping, T. Lancaster, S. J. Blundell, D. T. Adroja, V. Zapf, P. Gegenwart, and R. Coldea, Phys. Rev. B 99, 054426 (2019).

[32] N. P. Raju, E. Gmelin, and R. K. Kremer, Phys. Rev. B 46, 5405 (1992).

[33] J. A. Mydosh, Rep. Prog. Phys. 78, 052501 (2015).

[34] F. L. Pratt, J. Phys.: Condens. Matter 19, 456207 (2007). 